\title{
El Éxito en los Programas a Distancia: sus Egresados
}

\author{
(Success in Distance Education Programs: the Graduates)
}

\author{
José C. VÁzQueZ \\ Luisa A. NoA Silverio \\ Reina Reyna Figueroa
}

Facultad de Educación a Distancia. Universidad de La Habana

(Cuba)

RESUMEN: El presente artículo ofrece un análisis de la situación de los egresados de los programas en modalidad a distancia en la Facultad de Educación a Distancia de la Universidad de La Habana. Para ello se recurre a estudios realizados desde las primeras graduaciones: 1983-84, 1984-85 y 1985-86 hasta las últimas, incluyendo en ellos la graduación del curso 1996-97. Se presentan mediante cifras, las principales características de la población de graduados, los resultados de las encuestas aplicadas, así como las opiniones de los empleadores sobre el desempeño como profesionales de los egresados. En el próximo curso 1998-99, la educación a distancia en Cuba cumplirá sus veinte años de labor continuada, por ello es importante contribuir al conocimiento de este aspecto de su vida como institución educacional.

\section{Educación a Distancia - Egresado - Educación a Distancia en Cuba}

\begin{abstract}
The present paper is to provide an analysis on student graduated of distance education form of study in the programs developed at the Faculty of Distance Education of the University of Havana, Cuba. To meet these objectives previous researches which took into consideration from the first (1983-84) to 1996-97 promotions were consulted. The paper provides information on the characteristic of graduate population, principal survey data and the opinions of employers about performance of graduates in their new social role. In the next course 1998-99, the distances learning in Cuba will arrives its twentieth anniversary of continued work and growth in this field, and, therefor, it is an important goal to contribute to knowledge of these significant components of the education in our country.
\end{abstract}

\section{Distance Education - Graduate - Distance Education in Cuba}




\section{INTRODUCCIÓN}

En el inicio de la década de los años 80 (curso 1979-80) fue creada la Facultad de Enseñanza Dirigida, hoy Facultad de Educación a Distancia, lo que incluyó a nuestro país en la ola de surgimiento de centros en la modalidad a distancia que se había iniciado en el mundo entero desde principios de la década de los 70 . En el próximo curso 1998-99 la Facultad arribará a su XX Aniversario.

En esa década surgieron instituciones dedicadas sólo a la educación a distancia en el nivel superior de enseñanza, como fueron: La Open University de Gran Bretaña, la UNED (Universidad Nacional de Educación a Distancia) de España, la Athabasca University de Canadá, la UNA (Universidad Nacional Abierta) de Venezuela, la FernUniversität de Alemania, la UNED de Costa Rica, y de igual forma se crearon instituciones dedicadas a la educación a distancia en Pakistán, China, Israel, Irán, Japón, Tailandia y Países Bajos. La matrícula en todas estas instituciones fue y actualmente es del orden de miles y en algunos casos de cientos de miles. Otras instituciones de educación a distancia surgieron dentro de las universidades tradicionales como fue el caso de la Facultad de Enseñanza Dirigida de la Universidad de La Habana, que en su primer curso 1979-80 aceptó a más de 23 mil estudiantes.

¿Cuál fue la causa fundamental de todo este movimiento en el mundo entero que involucró tanto a los países desarrollados como los que se encuentran en vías de desarrollo? Esta es una pregunta que aún hoy, después de transcurridos cerca de 30 años, sigue siendo de interés teórico. En aquellos momentos la causa que se situaba en primer lugar era las ansias de superación de grupos que por circunstancias económicas, laborales y geográficas estaban marginados de las oportunidades educacionales. La apertura hacia nuevas o segundas oportunidades se basaban en la democratización de la enseñanza, en los fundamentos humanistas que esta apertura brindaba y su reflejo en la esfera de las decisiones políticas. Además, a estas tesis se le unió, sobre todo en nuestro continente, la idea de que por medio de la instrucción surgirían cambios en el estatus económico tanto en lo personal como en el país. Hoy en día los enfoques sobre las causas del surgimiento de la educación a distancia son más complejos y tienden a comprender numerosos factores como son los sociales en general, los referidos al desarrollo de las ciencias de la educación, los tecnológicos, los históricos y los factores propios de cada región, además de los ya señalados.

En el informe de la Comisión de Estudios no Tradicionales en Norte América, 1971 (MacKenzie, Postgate, Scupham, 1979, p.20) se señala que este tipo de estudio: “...pone al estudiante en primer término, y en segundo lugar, se interesa más por las necesidades de aquel que por la conveniencia de ésta (la institución); fomenta la diversidad de oportunidades individuales... y por ello quita importancia al tiempo, al espacio e incluso a los requisitos docentes, en favor de la com- 
petencia. Esta actitud... puede estimular un progreso educativo incitante y de gran calidad; pero si no se tiene cuidado en proteger la libertad que ofrece, puede ser involuntariamente un medio de disminuir el rigor académico e incluso de desembocar en el puro charlatanismo".

Desde esa última apreciación, sin esperar resultados y viendo más en la nueva modalidad sus defectos iniciales (falta de profesorado, profesorado joven, falta de locales, de materiales y de equipos, fuerte proporción de abandonos), la educación a distancia fue cuestionada y desestimada por los prejuicios de una parte de la comunidad académica. Pero ¿qué ha ocurrido en estos 30 años? ¿cuál es la realidad de la educación a distancia a finales del II Milenio?

La realidad presente es su expansión por el mundo entero; se calcula que no existe país alguno en que esta modalidad de estudio no tenga su expresión en algún programa o institución educativa. En educación a distancia es difícil dar un cuadro exacto porque los cursos y programas - desde los primeros niveles educacionales hasta los de nivel superior-son desarrollados tanto por instituciones educacionales públicas y privadas reconocidas como por medio de entrenamientos y cursos cortos ofrecidos por grandes y medianas empresas a sus trabajadores y personal de dirección. Un ejemplo de este crecimiento lo tenemos en Canadá, donde las cifras de hace dos años reportan que el 54\% de las universidades canadienses, el 68\% de los colegios comunitarios y el 36\% de las empresas grandes y de mediano tamaño en Canadá, reportan la utilización de la educación a distancia en sus programas educativos. (Roberts, 1996). Los pronósticos indican que dicha expansión continuará en los próximos años.

La potencialidad de la educación a distancia se refuerza, en nuestros días, por la inclusión de las nuevas tecnologías basadas en las comunicaciones mediadas por computadoras y los ambientes multimediales gráficos generados por ellas. Esta nueva vía de expansión de los estudios a distancia marcará profundamente su derrotero, a tal punto que cambiará las formas ya tradicionales de expresión de la propia educación a distancia, y promoverá el surgimiento de una nueva etapa en su desarrollo.

En nuestro país la educación a distancia se ha extendido a las universidades y a los centros universitarios del interior, de tal forma que en cada una de las 14 provincias en que se divide la Isla, se cuenta con posibilidades de estudio a distancia en cinco carreras: Contabilidad y Finanzas, Derecho, Historia, Economía e Información Científico Técnica y Bibliotecología.

Los primeros egresados de La Facultad de Educación a Distancia de la Universidad de La Habana, se graduaron en el curso 1983-84, a sólo 5 cursos de la creación de la Facultad. En ese curso obtuvieron sus títulos 20 estudiantes: 7 de Contabilidad y 13 de Derecho. Hoy, a casi veinte años de su curso inicial, y de contar 
con cerca de quince cursos de graduaciones sucesivas, en el pasado curso, 199697, se llegó a la cifra de más de MIL GRADUADOS mediante esta modalidad de estudio, solamente en la Universidad de La Habana. Los graduados de ese curso representan el $62 \%$ de todos los graduados del país en la modalidad a distancia.

Las instituciones de educación a distancia se caracterizan por su dinamismo y por el intercambio de experiencias entre ellas. De esta suerte se han creado numerosas asociaciones y se celebran anualmente conferencias, talleres, simposios, etc. Las ponencias a eventos y los trabajos en las publicaciones de educación a distancia están marcados por la atención priorizada al análisis de sus experiencias. Esta es una forma de contribuir al desarrollo de estudios teóricos y al esclarecimiento de sus tendencias mundiales. Aun hoy se sigue considerando que la base teórica en que se sustenta la educación a distancia es débil, sin que esto sea una reprobación ya que, comparada con las formas tradicionales universitarias surgidas en la Edad Media (Siglo XII y XIII), ésta es extremadamente joven. No obstante, en el mundo de hoy, las instituciones se transforman rápidamente debido a la aceleración de todos los procesos sociales y se hace necesario por esta razón, contribuir a su madurez mediante la reflexión sobre su práctica.

\section{LOS ESTUDIOS SOBRE LOS EGRESADOS DE LA EDUCACIÓN A DISTANCIA EN CUBA}

Los estudios emprendidos sobre los egresados de los sistemas de educación a distancia, abarcan desde la descripción meramente estadística hasta aquellos que involucran factores socioeconómicos.

El énfasis fundamental en las investigaciones sobre educación a distancia se realiza en direcciones tales como: la aplicación de los medios (radio, televisión, uso de ordenadores, vídeo, etc.), edición y publicación de textos, guías de estudios, problemas referidos a la enseñanza de adultos (tutorías, formas de estudio independiente, etc.) y la retención de matrícula o deserción, costo y en los últimos cinco años, la aplicación de las nuevas tecnologías a los programas a distancia ocupan un lugar preferente. Pocos trabajos encontramos que traten el éxito en los estudios y sí una amplia atención a la otra cara del problema: la deserción o abandono. En este sentido es pobre la referencia, aunque sólo se pretenda encontrar al menos algunas analogías y diferencias.

Los elementos sobre los egresados de los sistemas de educación a distancia se encuentran en general dentro de la esfera numérica, puramente estadística, en referencias en tablas y cifras, sin una descripción, salvo excepciones, de aspectos tales como los objetivos propuestos por los estudiantes, régimen de estudios, influencia del marco familiar y social, etc. La OPEN del Reino Unido ha realizado estudios sobre sus egresados que abarcan un gran número de aspectos, de igual 
forma, en estos momentos se realizan estudios de esta naturaleza en la Universidad de Educación a Distancia de España (UNED).

En nuestro país, la investigación sobre los egresados de esta modalidad se ha realizado dentro de la Facultad de Enseñanza Dirigida de la Universidad de La Habana, aunque graduados de esta modalidad existen en otras facultades del resto de las universidades y centros universitarios del país. Las graduaciones en la modalidad de estudios a distancia comprenden un 5\% a un 6\% de los egresados en centros de educación superior en el país.

Estos estudios comenzaron en el curso 1986-87, como parte del Tema de investigación: "El perfeccionamiento de los Cursos Dirigidos". Desde entonces, la investigación tenía el propósito de conocer las características de los egresados y sus experiencias como forma de obtener la mayor información posible para el perfeccionamiento y consolidación de los programas a distancia.

El primer estudio abarcó las tres primeras graduaciones: 1983-84, 1984-85 y 1985-86: (Colectivo de autores, 1989). Posteriormente se realizaron tres estudios más. El segundo abarcó los graduados de los cursos 1986-87 y 1987-88: (Noa, Noval, Chántez, Reyna, 1991). El tercero se realizó sobre los graduados de los cursos 1989-90 y 1990-91. Por último, el cuarto corte comprendió los graduados de tres cursos: desde 1991-92 hasta 1994-95: (Noa, Chántez, 1993), 1996).

La última de estas indagaciones busca conocer, además del estatus social alcanzado por los egresados en comparación con el que tenían antes de concluir sus estudios, su estado de satisfacción con el nivel de formación profesional obtenido y la percepción que de ello tienen los organismos empleadores y comprende los grupos de graduados de los cursos 199394, 199495 y 199596: (Vázquez, Reyna, 1996).

En el primer estudio, antes señalado para las tres primeras graduaciones, se aplicó una encuesta a los graduados con el objetivo, entre otros, de conocer su ubicación laboral antes y después de graduados; la segunda parte del cuestionario estaba dirigida a los jefes inmediatos superiores del graduado con el propósito de conocer la opinión sobre su grado de competencia. Los estudios siguientes no contemplaban este aspecto y se limitaban a obtener información de los graduados y sobre sus experiencias como estudiantes a distancia.

En el presente estudio que se detalla en este artículo, se aplicaron dos encuestas: una a los graduados y otra a los empleadores o jefes inmediatos superiores. Los objetivos propuestos eran similares a los de la primera encuesta señalada con anterioridad.

La encuesta a los graduados, además de obtener información de carácter general, se dirige a las formas de estudio, dificultades más importantes, utilización 
de los servicios que ofrece la Facultad, asesorías grupales, sala de lectura, grabación de cassettes de audio, etc.

La encuesta a los empleadores se dirigió, en lo fundamental, a conocer cómo se han ubicado los graduados en dichos puestos de trabajo, es decir, que vía fue utilizada, que opinión tienen de la preparación profesional del graduado y si existe alguna opinión de incluir determinados contenidos en los planes de estudios vigentes.

Para cumplimentar estos objetivos se analizaron un número de variables que propiciaban la obtención y procesamiento de la información de las fuentes siguientes:

Dos datos que constan en:

- Bancos de datos sobre los graduados de la Facultad.

- Archivo de la Facultad.

- Departamento de Estadística del Ministerio de Educación Superior (1997).

- Cuestionarios aplicados a una muestra de graduados y de empleadores.

D La encuesta aplicada que abarcó a 152 estudiantes distribuidos por carrera de la siguiente forma (Vázquez, Reyna, Noa, 1997):

- $78 \%$ de Derecho.

- $14 \%$ de Historia.

- $4 \%$ de Contabilidad y Finanzas.

- 3\% de Información Científico-Técnica y Bibliotecología.

- $1 \%$ de Economía.

\section{ANÁLISIS DE LA POBLACIÓN DE GRADUADOS}

\subsection{CARRERA}

Hasta el curso 1996-97 la cifra de graduados ascendió a 1 033. Como puede observarse de la tabla siguiente, en los últimos cuatro años se ha producido un incremento notable del número de graduados con relación a cursos anteriores, los que representan un promedio anual de 122 graduados en dicho período. En este incremento pueden incidir varios factores tales como: mayor cantidad de años de creada la Facultad de Enseñanza a Distancia lo cual permite que los primeros matriculados arriben a la conclusión de su carrera; la incorporación de estudiantes jóvenes sin vínculo laboral ni responsabilidades familiares, por lo que disponen de mayor tiempo para incorporarse al estudio, entre otros. 
El Éxito de los Programas a Distancia: sus Egresados

Tabla 1. Graduados por Carrera

\begin{tabular}{|l|r|r|r|r|r|r|r|}
\hline CURSO & CONT & DER & HIST & PEN & ICTB & ECO & Totales \\
\hline $1983-84$ & 7 & 13 & & & & & 20 \\
\hline $1984-85$ & & 37 & 7 & & & & 44 \\
\hline $1985-86$ & 1 & 32 & 17 & & & & 50 \\
\hline $1986-87$ & 5 & 25 & 6 & & & & 36 \\
\hline $1987-88$ & 2 & 47 & 12 & & & & 61 \\
\hline $1988-89$ & 4 & 75 & 13 & 2 & 1 & & 95 \\
\hline $1989-90$ & 2 & 50 & 7 & 1 & 4 & & 64 \\
\hline $1990-91$ & 1 & 27 & 10 & & 6 & & 44 \\
\hline $1991-92$ & & 42 & 13 & & 3 & 2 & 60 \\
\hline $1992-93$ & 1 & 50 & 14 & & 6 & & 71 \\
\hline $1993-94$ & 5 & 81 & 14 & & 10 & 3 & 113 \\
\hline $1994-95$ & 4 & 94 & 15 & & 14 & 8 & 129 \\
\hline $1995-96$ & 2 & 75 & 20 & & 15 & 2 & 114 \\
\hline $1996-97$ & & 106 & 17 & & 9 & & 132 \\
\hline TOTALES & 34 & 754 & 165 & 3 & 58 & 15 & 1033 \\
\hline
\end{tabular}

CONT Contabilidad y Finanzas.

DER Derecho.

HIST Historia.

PEN Planificación de la Economía Nacional (sustituida por la carrera de Economía).

ICTB Información Científico-Técnica y Bibliotecología.

ECO Economía.

(En esta tabla no aparecen recogidos 4 estudiantes graduados de Ciencias Sociales en la República de Cabo Verde).

En relación con la matrícula, los estudiantes de la carrera de Derecho que hasta el curso 1994-95 constituían una cifra superior al 67\%, muestran un ligero descenso en los dos últimos años ya que en el curso 1995-96 alcanzaron el 65,6\% En el curso 1996-97 el por ciento de matriculados fue del 63,4.

El mayor número de graduados lo tiene la carrera de Derecho con un $73 \%$ del total. El segundo lugar, por su contribución a la cifra de graduados, lo tiene la carrera de Historia, con un 16\%, la carrera donde menos estudiantes se gradúan es la de Economía.

Como marco de referencia señalaremos que la matrícula en la Facultad en el primer curso: 1979-80 alcanzó más de 23 mil estudiantes, entre los cursos 82-83 hasta 89-90, ésta fue de más de 11 mil estudiantes. El curso siguiente 1990-91 y 
coincidiendo con el Período Especial por el que ha atravesado el país, la matrícula fue descendiendo hasta alcanzar su nivel más bajo en el curso 1995-96 con 6062 estudiantes. A partir de este curso ha comenzado a aumentar el número de matriculados, y en el curso actual se cuenta con casi 8 mil estudiantes. Esta situación no se ha dejado sentir en el número de graduados que como puede verse en la Tabla No. 1, la tendencia ha sido hacia su incremento.

\subsection{SEXO}

La matrícula en la Facultad por sexo hasta el curso 1986-87 mostraba una mayoría del sexo masculino; después de este curso la diferencia entre ambos sexos se fue reduciendo paulatinamente hasta igualarse en los cursos 1993-94 y 1994-95. A partir del curso siguiente la matrícula de mujeres empieza a superar a los hombres. En el curso 1997-98, las mujeres representan un 55\% de la matrícula.

Esta situación también comienza a reflejarse en los graduados. Así tenemos que hasta el curso 1994-95 las mujeres sólo representaban una tercera parte de los que culminaban estudios, proporción ésta que era inferior a la de la matrícula. Es por ello que en el estudio realizado sobre estos grupos, se señalaba que la tendencia en la matrícula era a igualar las cifras entre hombres y mujeres, mientras que en la población de graduados en general el 33\% era del sexo femenino, es decir, sólo una tercera parte de los graduados eran mujeres, lo que evidenciaba un desplazamiento hacia el sexo masculino en cuanto a la obtención del título. Pero ya en los cursos 1995-96 y 1996-97 las mujeres representaron el 51\% y $58 \%$ de los graduados, como puede verse en la siguiente tabla.

Tabla 2. Graduados por sexo y curso académico

\begin{tabular}{|l|c|c|c|c|}
\hline CURSO & FEMEN. & $\%$ & MASCUL. & $\%$ \\
\hline $1991-92$ & 20 & 33 & 40 & 67 \\
\hline $1992-93$ & 20 & 28 & 51 & 72 \\
\hline $1993-94$ & 43 & 38 & 70 & 62 \\
\hline $1994-95$ & 37 & 29 & 92 & 71 \\
\hline $1995-96$ & 58 & 51 & 56 & 49 \\
\hline $1996-97$ & 76 & 58 & 56 & 42 \\
\hline
\end{tabular}

A esto ha contribuido el hecho de que en la matrícula de los últimos cursos se ha incrementado el número de las mujeres jóvenes. En los cursos regulares (educación presencial) ocurre una situación similar: en el curso 1976-77 las mujeres constituían el $40 \%$ de la matrícula en la educación superior, en estos momentos la situación se ha invertido, para ocupar las mujeres el $60 \%$ de esta matrícula. 
El Éxito de los Programas a Distancia: sus Egresados

\subsection{EDAD}

A partir del curso 1989-90 empezó a hacerse evidente la tendencia en la matrícula hacia una población estudiantil cada vez más joven. Si en los dos primeros años de creada esta modalidad de estudio los matriculados que tenían 30 años o más representaron el 52\% y 66\% respectivamente, en el curso 1996-97 este grupo de edades sólo fue del $31 \%$, incrementándose las cifras de edades por debajo de 30 años.

Esta situación empieza a reflejarse también en los estudiantes que culminan sus estudios. Así en el curso 1996-97 el 53\% de los graduados tienen menos de 30 años.

En relación con la muestra de graduados estudiados, la edad promedio de los mismos es de 37 años.

La correlación entre edad y sexo respecto a los estudiantes que asisten a exámenes es también interesante. En una encuesta aplicada en el curso 1996-97 a 953 estudiantes se obtuvo lo siguiente: a los exámenes acuden una proporción mayor de mujeres que de hombres, proporción ésta que no se corresponde con la relación entre hombres y mujeres que guarda la matrícula. Los estudiantes que acuden a examen con edades entre 18 y 25 años es muy superior a la representatividad que estas edades tienen en la matrícula y de igual forma se comportan los trabajadores. Como es natural, en un período corto de tiempo estos hechos se reflejan en la obtención de títulos.

\subsection{CLASE DE ESTUDIANTES}

Bajo este indicador se enmarcan los estudiantes según su ocupación laboral. Los estudios a distancia, en nuestro país, surgieron para dar respuesta a la demanda de los trabajadores que deseaban continuar su superación incorporándose a programas en el nivel universitario, por lo que en los primeros años la masa fundamental de estudiantes estaba compuesta por trabajadores. Una muestra de ello lo tenemos en los cursos 1979-80 y 1980-81 donde las cifras de trabajadores constituían el $89 \%$ y 93\% de la matrícula respectivamente.

Al establecerse un límite al número de estudiantes que pueden ingresar a la enseñanza superior, se produce un incremento en la cifra de egresados de la enseñanza media superior que no alcanzan plazas para estudiar en los centros universitarios, lo que provoca que éstos acudan a matricular en la modalidad a distancia en un número cada vez mayor. En consecuencia disminuye la edad promedio de nuestros estudiantes y la proporción del número de trabajadores que matriculan. Por ejemplo en el pasado curso académico la cifra de trabajadores matriculados solo alcanzó el 51\%. 
En relación con la muestra de graduados encuestados la situación es como sigue:

$80 \%$ trabajadores estatales

$2 \%$ trabajadores por cuenta propia

$18 \%$ no trabajan

\subsection{NÚMEROS DE CURSOS}

Los estudiantes a distancia emplean un promedio de un poco más de 6 cursos para graduarse en sus respectivas carreras. La tendencia es a la disminución del número de cursos empleados para culminar los estudios. En las Facultades que emplean las formas tradicionales de enseñanza, estos programas se desarrollan en cinco cursos.

TABLA NO. 3

Cantidad de graduados por curso académico y por números de cursos empleados para su graduación $(\%)$

\begin{tabular}{c|c|c|c|c|c|c}
\hline $\begin{array}{c}\text { Números de } \\
\text { cursos }\end{array}$ & $\begin{array}{c}\text { Curso } \\
83-84\end{array}$ & $\begin{array}{c}\text { Curso } \\
86-87\end{array}$ & $\begin{array}{c}\text { Curso } \\
89-90\end{array}$ & $\begin{array}{c}\text { Curso } \\
92-93\end{array}$ & $\begin{array}{c}\text { Curso } \\
95-96\end{array}$ & $\begin{array}{c}\text { Curso } \\
96-97\end{array}$ \\
\hline 2 & & 0 & 3 & 1 & 3 & \\
\hline 3 & 4 & 3 & 6 & 1 & 9 & 2 \\
\hline 4 & 20 & 11 & 3 & 4 & 19 & 14 \\
\hline 5 & 76 & 3 & 3 & 15 & 11 & 26 \\
\hline 6 & & 24 & 16 & 12 & 12 & 11 \\
\hline 7 & & 14 & 17 & 15 & 19 & 11 \\
\hline 8 & & 46 & 13 & 10 & 6 & 11 \\
\hline 9 & & & 13 & 13 & 3 & 8 \\
\hline 10 & & & 6 & 8 & 5 & 3 \\
\hline 11 & & & 20 & 3 & 4 & 1 \\
\hline 12 & & & & 3 & 4 & 4 \\
\hline 13 & & & & 5 & 3 & 1 \\
\hline 14 & & & & 13 & 1 & \\
\hline 15 & & & & 5 & 1 & 2 \\
\hline 16 & & & & & & \\
\hline 17 & & & & & 1 & \\
\hline 18 & & & & & & $* *$ \\
\hline
\end{tabular}


Los graduados del curso 1992-93 emplearon como promedio 7.5 cursos; los de 1995-96, 6.7 y los de 1996-97 emplearon 6.9 cursos.

* Se han eliminado de la representación en la tabla los estudiantes que provienen como trasladados de la modalidad presencial, con más del $90 \%$ de las asignaturas aprobadas por dicha modalidad.

** En esta categoría tenemos un graduado que había matriculado por primera vez en el curso 1979-80, es decir, que se graduó 18 años después. Muchos estudiantes matriculan y dejan por un tiempo sus estudios en la modalidad a distancia, más tarde reingresan a la Facultad y pueden culminar sus estudios.

\section{RESULTADOS MÁS IMPORTANTES DE LA ENCUESTA APLICADA}

\subsection{FACTORES MOTIVACIONALES}

Según los graduados encuestados las principales motivaciones que los llevaron a matricular en esta modalidad de estudios fueron:

96\% Superarse y elevar su nivel cultural.

$81 \%$ Tener inclinación, vocación hacia la carrera.

$80 \%$ Sentirse más útil socialmente.

$61 \%$ Obtener un título universitario.

El factor económico "obtener una promoción y mejorar ingresos" muestra un incremento de un 10\% en relación con las encuestas aplicadas con anterioridad en otros estudios.

En el estudio realizado con los graduados de los cursos 198990 y 199091, se obtuvo los siguientes resultados sobre estos aspectos:

“Elevación del nivel cultural y la superación.

Este factor alcanzó el $94 \%$ de las respuestas en la escala más alta de valoración. (Mucho, regular, poco, nada) Otras consideraciones importantes fueron:
Sentirse útil socialmente
Por vocación o inclinación
$(73 \%)$
Continuar estudiando
$(64 \%)$

Los factores económicos o vinculados con promociones laborales tienen un segundo plano:

Búsqueda de un nivel de ingreso superior

Promoción laboral

Cambio de profesión 
La conservación del empleo como factor impulsor de sus metas en el estudio, es considerada como muy poco influyente, el $54 \%$ lo señaló en la escala de "nada influyente".

Estas últimas cifras coinciden con los criterios de los graduados actuales y sólo varían al dar estos últimos un mayor peso al factor económico.

Las motivaciones y necesidades de superación se manifiestan también en estas encuestas por la solicitud expresa que hacen los graduados encuestados de que se le ofrezcan, por esta modalidad de estudios, cursos de postgrado. Así en el segundo estudio realizado sobre los graduados (Noa, Noval Chántez, Reyna, 1991):

El 81\% se interesaba por participar en algún curso de postgrado organizado por la Facultad. El 100\% de las carreras de Historia y Contabilidad solicitaron esta forma de superación, es decir, cursos de postgrado a distancia.

El presente estudio arroja que un $85 \%$ de los graduados encuestados solicita que se le ofrezcan posibilidades de superación a distancia.

\subsection{FORMAS DE ESTUDIO}

En cuanto a las formas de estudio utilizadas, la gran mayoría (97\%) planteó que lo hizo siempre o casi siempre de forma individual; sólo el 22\% manifestó haber consultado con profesores y de ellos, el 9\% dice haberlo hecho siempre. El $12 \%$ dice haber consultado siempre o casi siempre con otros estudiantes.

El 50\% manifiesta que estudia en bibliotecas siempre o casi siempre; el 88\% realiza sus estudios en el hogar. Solamente el 27\% manifiesta que estudió en colectivo: siempre (8\%) y casi siempre (19\%).

El 76\% dice que planificó regularmente las asignaturas a examinar por el orden de precedencia y ciclos y el $67 \%$ que lo hizo regularmente por contar con la bibliografía.

La asistencia a las asesorías de los metodólogos o profesores para planificar las asignaturas a examinar es muy baja, el 11\% y 13\% respectivamente.

El 44\% utilizó sistemáticamente las oportunidades de orientación que le brinda el Centro, el $50 \%$ de los que no usaron este servicio dijeron desconocer que existía.

En relación con los materiales de apoyo más utilizados, el 29,6 dice que usa los resúmenes de otros compañeros; el 16\% que usa resúmenes propios y el 15,7\% dice haber asistido a conferencias de profesores. 
En relación con las sugerencias que se hacen, el 19,7\% manifiesta la necesidad que se dé solución a la falta de bibliografía; el 15,7 plantea que se ofrezcan asesorías de contenidos y el 8,5 que se debe agilizar el tiempo y mejorar el sistema de calificaciones.

\subsection{SATISFACCIÓN Y CALIDAD}

El 93\% de los graduados encuestados plantean que alcanzaron su objetivo de "superarse y elevar su nivel cultural" y el $82 \%$ dice "sentirse más útil socialmente"; mientras que el $42 \%$ plantea haber "obtenido una promoción laboral y mejorar ingresos", en tanto el 39\% dice que "cambió de empleo o profesión".

En relación con los cambios experimentados después de graduarse el $82 \%$ se siente más eficiente y capaz y el 75\% tiene más confianza en sí mismo; el 54\% plantea que mejoró su estatus laboral.

El 52\% asumió determinadas responsabilidades en su centro de trabajo al graduarse y de ellos el $41 \%$ califica esta responsabilidad con el nivel máximo.

El 25\% se declara satisfecho con lo logrado al graduarse y el 65\% dice estar muy satisfecho.

El 83\% plantea que los conocimientos adquiridos le han sido útiles para su empleo.

El 77\% expresa que se siente en iguales condiciones que los graduados de los cursos presenciales.

Si contrastamos estos resultados con la encuesta aplicada a los empleadores veremos que el $98 \%$ considera que los graduados mediante esta modalidad de estudios poseen los conocimientos necesarios para desempeñar las funciones que le son asignadas y el 88\% manifiesta que si tuvieran la oportunidad de elegir escogería a estos graduados para el desempeño de un puesto de trabajo.

Resultado muy similar se obtuvo en la primera encuesta a empleadores. En este primer informe se subrayaba lo siguiente:

"Dada la forma de estudio de este tipo de enseñanza basada en la autopreparación, el estudiante por sí mismo, necesariamente tiene que desarrollar una capacidad de organización de sus estudios, de planificación de su tiempo, de sentido crítico y autocrítico, de toma de decisiones. Esto lo logra por mecanismos muy variados siguiendo el método de ensayoerror, como por ejemplo, solicitando por su cuenta materiales de otros tipos de curso, o asistiendo a consultas programadas para otros estudiantes, etc. Ello trasciende el marco de su ser como estudiante y se amplía y concreta tanto en su vida individual como laboral. 
Así ha sido valorado por los jefes inmediatos superiores de estos graduados quienes, además, consideran que el centro de trabajo recibe pronta y continuamente tales beneficios, por cuanto el trabajadorestudiante tiene ya una determinada disciplina de trabajo y utiliza a tiempo completo su jornada laboral en el centro. A todo esto debe agregarse que muchos de los graduados de Derecho y en parte, los de Contabilidad, han estado vinculados a actividades afines con el perfil profesional que eligieron estudiar.

Asimismo, la preparación docente recibida los ha capacitado para desempeñar eficazmente las funciones relativas a la plaza que ocupan, las cuales dominan profesionalmente.

El hecho de que estos grupos de graduados de la Facultad estaban vinculados con actividades afines a la carrera que estudiaban, garantiza que al menos los elementos prácticos y ejercitación mínima necesarias se logren por esta vía, así como el vínculo con grupos con una determinada "cultura" en estas ramas. Pero no es posible olvidar que la mayoría de los estudiantes no tienen ese ambiente ideal."

A la hora de caracterizar a los graduados de educación a distancia, en la reciente encuesta, los empleadores lo hacen de la forma siguiente:

- $92 \%$ cumplidores (43\%) y muy cumplidores (49\%).

- $92 \%$ estudiosos (37\%) y muy estudiosos (55\%).

- $88 \%$ organizados (51\%) y muy organizados $(37 \%)$.

- $86 \%$ independientes (35\%) y muy independientes (51\%).

- $86 \%$ creativos $(53 \%)$ y muy creativos $(33 \%)$

- $82 \%$ sistemáticos (39\%) y muy sistemáticos (43\%).

- $78 \%$ habilidosos (43\%) y muy habilidosos $(35 \%)$.

- $76 \%$ conocedores $(43 \%)$ y muy conocedores $(33 \%)$.

- $70 \%$ esforzados $(39 \%)$ y muy esforzados $(31 \%)$.

- $68 \%$ emprendedores (37\%) y muy emprendedores $(31 \%)$.

El 35\% de los empleadores coinciden en plantear que los estudiantes debían realizar prácticas laborales, lo que propiciaría una mejor preparación.

\section{CONCLUSIONES}

La extensión de estos estudios sobre graduados comprende todas las promociones de graduados, es decir, que no se ha dejado ni un solo curso donde no se haya obtenido una muestra representativa y se haya aplicado la encuesta bajo parámetros similares que permitan hacer una valoración de las posibles tendencias. En estos estudios se analizan cuestiones muy específicas del sistema como son las dificultades con determinadas asignaturas, que como es comprensible, por el alcance de la publicación, no exponemos al lector. 
El Éxito de los Programas a Distancia: sus Egresados

Los graduados de programas a distancia, ya sean terminales como en nuestro caso o no, fomentan hábitos de estudio donde la autogestión es el eje fundamental, así como hábitos y disciplina de organización muy útiles para desarrollar las tareas propias de su profesión. Estos elementos han sido apreciados, tanto por los empleadores como por el propio estudiante. En una de estas encuestas se le pedía a los graduados sus opiniones sobre el estudio a distancia y los estudios tradicionales. He aquí algunas de estas respuestas:

- Consideraban que los estudiantes a distancia estaban mejores preparados teóricamente.

- En su caso particular considera que desde su puesto de trabajo adquirió el complemento práctico para su funciones después de graduado.

- Para los graduados de estudios a distancia son necesarios cursos de postgrado, seminarios, etc. No se puede obviar el hecho de que los estudiantes del curso regular mantienen un contacto directo con sus profesores, los cuales en el desarrollo de las clases, les transmiten conocimientos, experiencias y vivencias al margen de los textos... lo que resulta insustituible como método de enseñanza.

- Tenía 16 años de experiencia en la actividad cuando matriculé, al finalizar contaba con 26 años de experiencia. Esto es una gran ventaja sobre los egresados de los cursos regulares.

- El estudio de forma autodidacta tiene sus desventajas.

- Los egresados de los cursos regulares son más jóvenes y tienen más oportunidades sociales.

- Los materiales de estudio con que contamos no nos permiten suprimir la desventaja.

En estas respuestas brindadas por los graduados se continúa considerando algunas de las desventajas del estudio a distancia pero consideran la necesidad de utilizar otras formas y no copiar los métodos utilizados en la enseñanza presencial.

Por todas estas razones ya la Facultad ha iniciado la preparación de un pilotaje para instrumentar la práctica pre-profesional en aquellos estudiantes próximos a realizar el Examen Estatal y se realiza un esfuerzo en brindarles materiales escritos como las guías de estudio y orientaciones a través de los audiocasetes. Porque, si estos elementos lo aportan los que ya se han graduado, seguramente será de gran ayuda a aquellos que aún no lo han hecho.

Estudios como éste nos indican que la educación a distancia, de forma indiscutible, se ha ganado un espacio en nuestra sociedad y que a través de ella muchas personas han podido estudiar y alcanzar su titulación con una buena acep- 
tación por parte de los que utilizan los servicios de estos profesionales. En Cuba el 5\% de la población ha culminado estudios universitarios, y aunque pequeño, el aporte de la modalidad a distancia no es despreciable.

\section{REFERENCIAS BIBLIOGRÁFICAS}

1. Colectivo de Autores (1989). Informe Interno sobre Estudio sobre Egresados de la Facultad de Enseñanza Dirigida.

2. Departamento de Estadística del Ministerio de Educación Superior. Datos aportados sobre los cursos de 1995-96 y 1996-97.

3. MacKenzie, N., Postgate, R., Scupham, J. (1979). Enseñanza Abierta. Sistemas de enseñanza postsecundaria a distancia. Madrid: UNESCO.

4. Noa, L., Noval, E., Chántez,S., Reyna, R. (1991). Estudio sobre los graduados. En Memorias del I Taller Internacional de Educación a Distancia. La Habana.

5. Noa, L., Chántez, S. (1993). Estudio sobre los Egresados de las Carreras de Enseñanza Dirigida. En Memorias del II Taller Internacional de Educación a Distancia, celebrado en La Habana.

6. Roberts, J. (1996). The Story of Distance Education: A Practitioner's Perspective. Journal of the American Society for Information Science. 47(11). John Wiley and Sons. Inc. 811-816.

7. Vázquez, J., Reyna, R. (1996). Estudio sobre los Graduados de la Facultad de Enseñanza Dirigida. (Ponencia) III Taller Internacional de Educación a Distancia, celebrado en La Habana.

8. Vázquez, J., Reyna, R., Noa, L. (1997). Informe sobre el resultado de la Encuesta aplicada a los Estudiantes de Educación a Distancia (Facultad de Educación a Distancia, Universidad de La Habana). Informe Interno.

\section{PERFIL ACADÉMICO-PROFESIONAL DE LOS AUTORES}

José Carlos Vázquez López. Profesor de Lógica. Ha participado en los estudios realizados sobre los graduados. Actualmente investiga en la esfera de la elaboración de materiales escritos. Desempeña el cargo de Vicedecano de la Facultad de Educación a Distancia de la Universidad de La Habana.

Facultad de Educación a Distancia

Universidad de La Habana

Edificio Varona

San Lázaro y Calle L

Vedado. CP 10400

Hab. 4. Cuba.

Teléfono: (537) 781112

FAX: (537) 335773

Correo Electrónico: fed@comuh.uh.cu

Luisa Noa Silverio. Profesora Titular. Ha participado en los estudios realizados sobre los graduados. Investiga desde hace algunos años en la incorporación, de forma experimental, de las aplicaciones multimedia a los programas a distancia. Responsable del Grupo de Tecnología Educativa.

Facultad de Educación a Distancia 
Universidad de La Habana

Edificio Varona

San Lázaro y Calle L

Vedado. CP 10400

Hab. 4. Cuba.

Teléfono: (537) 781112

FAX: (537) 335773

Correo Electrónico: luisaoa@comuh.uh.cu

Reina Reyna Figueroa. Profesora de Historia. Ha participado en los estudios realizados sobre los graduados. Actualmente investiga en la esfera de la elaboración de materiales escritos. Responsable del Grupo de Materiales Escritos y Logística.

Facultad de Educación a Distancia

Universidad de La Habana

Edificio Varona

San Lázaro y Calle L

Vedado. CP 10400

Hab. 4. Cuba.

Teléfono: (537) 781112

FAX: (537) 335773

Correo Electrónico: fed@comuh.uh.cu 